formed in intact red cells or are an artefact of membrane preparation.

Band 2.1 and its progeny have been variously named ankyrin (Greek, anchor), nectin (Latin, to connect), and syndein (Greek, to bink together). Ankyrin was chosen first, but because this name had already been given to a plant protein and because it soon became clear that ankyrin was not the true anchor (see below), the protein was rechristened nectin. Unfortunately this name was also a duplicate, so a second change to syndein was made. Some of those working in the field continue to use ankyrin while others have adopted the revised terminology. Presumably the rest of us will have to choose between these appellations (I prefer ankyrin) or make up our own, at least until some Commission on Nomenclature decrees.

In the latest sequel to this saga (Nature 280, 468; 1979), Bennett and Stenbuck report that ankyrin is firmly linked to band 3 in detergent extracts of spectrin-depleted red cell membranes. (It should be noted that some band 4.2 is also associated with band 3 in these extracts. The data presented favour the author's interpretation of a direct linkage between ankyrin and band 3 , but do not completely exclude the possibility that band 4.2 is interposed between these two proteins). The band 3 molecules involved $(10-20 \%$ of the total) are apparently identical to the principal band 3 protein which functions as an anion exchange channel. Unlike ankyrin this protein spans the lipid bilayer and is presumably the true site of skeletal anchorage. Ankyrin is thus not an anchor but an anchor chain - a protein that links spectrin and band 3 . In fact it may function generally to connect cytoskeletal elements and integral membrane poteins since, unlike spectrin (see below), it is apparently present in many types of cells (Bennet \& Stenbuck op. cit.).

The electron microscopic techniques used to visualise the band 4.1 binding site have also been applied to ankyrin (Tyler \& Branton op. cit.) and are even more successful since ankyrin is a larger molecule (a $80 \AA \times 95 \AA$ pyramid) than band 4.1 (a $60 \AA$ sphere). These studies clearly show that ankyrin binds to a site $200 \dot{\mathrm{A}}$ distal to the end of spectrin involved in the dimertetramer interaction. The stoichiometry of this linkage (whether there are one or two ankyrins per tetramer) remains to be determined.

\section{Membrane skeletal model}

Taken together, this information provides a fairly clear picture of how the principal skeletal proteins are probably organised (Fig. 1). Certain general points remain to be established (for example, the stoichiometry of the spectrin-actin-band 4.1 and spectrin-ankyrin-band 3 complexes and the physical state of actin), and many structural details must still be pursued, but the general framework appears to be taking shape. It must be emphasised however,

\title{
Halley's Comet in ancient history
}

from David $W$. Hughes

RECENT books on comets state categorically that the first definitely recorded return of Halley's comet was that of $240 \mathrm{BC}$, some adding as a postscript that there is a vague possibility that comets described in $613 \mathrm{BC}$ and $467 \mathrm{BC}$ may also have been $\mathrm{P} /$ Halley (the $\mathrm{P} /$ prefix indicating a periodic comet). It now seems, however, that observations go back considerably further. Y. C. Chang of the Purple Mountain Observatory, Nanking, China finds that the earliest astronomical record of the comet in the historical annals of his country described the apparition of 1057 BC. Reasons for this assertion, together with other early Chinese observations, are published in Chang's recent paper in Chinese Astronomy (3, 120; 1979) (a translation of Acta Astr. Sinica 19, 109; 1978).

The problem of recognising the historical record as a description of Halley's comet has been approached in two ways. The first relies on celestial mechanics to produce an accurate analysis of the orbital evolution of the comet. Chang started with the position and velocity vectors of Halley's comet and the eight major Solar System planets for a specified epoch (Julian day 2430000.5). The cometary results were taken from Brady and Carpenter (Astr. $J$ 76,$728 ; 1971$ ) who based their findings on 3085 observations of $\mathrm{P} / \mathrm{Halley}$ made by 60 observatories over an unbroken period of 20 months during the 1910 return.

The equation of motion of the comet around the Sun was then integrated, taking into account gravitational perturbations by the planets. This enabled the perihelion passage times and orbital elements of $\mathrm{P} / \mathrm{Halley}$ to be established for the previous 3000 years. So Chang knew when and where in the sky the comet would have appeared. The planetary perturbations produce changes in the orbital period of the comet, which was for example about 72.9 years between $762 \mathrm{BC}$ and $689 \mathrm{BC}$, and as long as 79.7 years between $A D 1222$ and $A D 1301$. The inclination of the orbit has decreased by about $1.5^{\circ}$ over 3000 years, and during this time the argument of perihelion has changed from $77^{\circ}$ to $112^{\circ}$ and the longitude of the descending node from $18^{\circ}$ to $58^{\circ}$. A more important parameter is the maximum brightness of the comet.

that the true skeleton is undoubtedly more complicated than the model shown in Fig 1. Skeletal proteins like bands $4.2,4.9$ and 7 are not included in the model since too little is known about their location and function. Band 4.1 must attach to the bilayer in some way since most of it remains on the membrane when spectrin and actin are extracted at low ionic strength. And
This seems to change by about 2.7 magnitudes per millennium, indicating that $\mathrm{P} /$ Halley will reach a maximum magnitude about +0.5 at its next apparition in 1986, whereas it was about -7.7 in $1057 \mathrm{BC}-$ brighter than any of the planets.

Brady (Pub. Astr. Soc. Pacific 83, 314 ; 1972) also studied the orbital evolution of $\mathrm{P} /$ Halley and suggests that the present planets could not account for all the observed changes. To overcome this difficulty he predicted the existence of another planet at a distance of 64 astronomical units (AU) from the Sun, with a mass three times that of Saturn. Chang does not confirm this hypothesis and finds that the known planets account for the observed perturbations. Some astronomers have also proposed that there is a cloud of comets about $50 \mathrm{AU}$ from the Sun having a total mass similar to that of the Earth. Even though $\mathrm{P} / \mathrm{Halley}$, with its aphelion distance of 35 $A U$, would spend a considerable time near this cloud, Chang again finds that such a cloud is unnecessary when it comes to accounting for the perturbation of $\mathrm{P} /$ Halley.

Chang's second approach was to peruse the Chinese astronomical records, the most complete historical records in existence, for accounts of Halley's comet. The new account came to light in a chapter on military strategy in the Book of Prince Huai Nan. This recounts how a comet was seen in the east with its tail pointing to the northwest at the time when King Wu marched on Zhou of Yin. The book also gives the position of Jupiter at that time. There are three schools of historical thought about the time of Wu's campaign, favouring $1122 \mathrm{BC}$, 1075-1055 BC or 1027 BC. All agree however, that it occurred in the first two months after the winter solstice. By combining these factors Chang concluded that the campaign and the observation of Halley's comet took place in the winter of $1058 / 57 \mathrm{BC}$, so our next observations of $\mathrm{P} /$ Halley in $1985 / 86$ will not be the thirtieth, as previously thought, but the forty-first recorded apparition.

David W. Hughes is a Lecturer in the Department of Physics, University of Sheffield.

spectrin chains must often cross each other since the average spacing between ankyrinlinked band 3 dimers $(\sim 375-530 \AA)$ is considerably less than the average length of a spectrin tetramer $(\sim 2000 \mathrm{~A})$.

\section{Phosphorylation}

Most of the membrane skeletal proteins 